\title{
ON THE STABILITY OF A MIXED-TYPE LINEAR AND QUADRATIC FUNCTIONAL EQUATION
}

\author{
PAISAN NAKMAHACHALASINT
}

(Received 7 June 2007)

\section{Abstract}

We give the general solution of the $n$-dimensional mixed-type linear and quadratic functional equation,

$$
\left(\begin{array}{c}
n-2 \\
m-2
\end{array}\right) f\left(\sum_{i=1}^{n} x_{i}\right)+\left(\begin{array}{c}
n-2 \\
m-1
\end{array}\right) \sum_{i=1}^{n} f\left(x_{i}\right)=\sum_{\left\{i_{1}, \ldots, i_{m}\right\} \in P_{m}} f\left(\sum_{k=1}^{m} x_{i_{k}}\right),
$$

where $P_{m}=\{A \subset\{1,2, \ldots, n\}:|A|=m\}$, and $1<m<n$ are integers.

2000 Mathematics subject classification: 39B22, 39B52, 39B82.

Keywords and phrases: functional equation, mixed-type functional equation, stability.

\section{Introduction}

In 1940 Ulam [6] proposed the famous Ulam stability problem of linear mappings. In 1941 Hyers [2] considered the case of approximately additive mappings $f: E \rightarrow E^{\prime}$ where $E$ and $E^{\prime}$ are Banach spaces and $f$ satisfies the inequality $\| f(x+y)-f(x)-$ $f(y) \| \leq \varepsilon$ for all $x, y \in E$. It was shown that the limit $L(x)=\lim _{n \rightarrow \infty} 2^{-n} f\left(2^{n} x\right)$ exists for all $x \in E$ and that $L: E \rightarrow E^{\prime}$ is the unique additive mapping satisfying $\|f(x)-L(x)\| \leq \varepsilon$. Rassias [5] generalized the result to the case when the inequality is controlled by the sum of norms. Since then, the stability problem has been investigated for various functional equations.

Rassias [4] established the Ulam stability of the following mixed-type functional equation:

$$
f\left(\sum_{i=1}^{3} x_{i}\right)+\sum_{i=1}^{3} f\left(x_{i}\right)=\sum_{1 \leq i<j \leq 3} f\left(x_{i}+x_{j}\right) .
$$

The present author [3] generalized the above functional equation to the following $n$-dimensional functional equation:

$$
f\left(\sum_{i=1}^{n} x_{i}\right)+(n-2) \sum_{i=1}^{n} f\left(x_{i}\right)=\sum_{1 \leq i<j \leq n} f\left(x_{i}+x_{j}\right) .
$$

(C) 2008 Australian Mathematical Society 0004-9727/08 \$A2.00 + 0.00 
In this paper, we will further generalize the above equation to

$$
\left(\begin{array}{l}
n-2 \\
m-2
\end{array}\right) f\left(\sum_{i=1}^{n} x_{i}\right)+\left(\begin{array}{c}
n-2 \\
m-1
\end{array}\right) \sum_{i=1}^{n} f\left(x_{i}\right)=\sum_{\left\{i_{1}, \ldots, i_{m}\right\} \in P_{m}} f\left(\sum_{k=1}^{m} x_{i_{k}}\right),
$$

where $1<m<n$, and we will investigate its generalized stability.

Throughout the paper, we denote the dimensionality of the problem by $n$, and let $P_{m}=\{A \subset\{1,2, \ldots, n\}:|A|=m\}$. Moreover, we use subscripts $e$ and $o$ to denote the even part and the odd part of a function, respectively. The even part of a function $f$ is defined by

$$
f_{e}(x)=\frac{f(x)+f(-x)}{2},
$$

and the odd part of $f$ is defined by

$$
f_{o}(x)=\frac{f(x)-f(-x)}{2} .
$$

\section{The general solution}

THEOREM 1. Let $1<m<n$ be integers, and let $X$ and $Y$ be vector spaces.

A function $f: X \rightarrow Y$ satisfies the functional equation

$$
\left(\begin{array}{c}
n-2 \\
m-2
\end{array}\right) f\left(\sum_{i=1}^{n} x_{i}\right)+\left(\begin{array}{c}
n-2 \\
m-1
\end{array}\right) \sum_{i=1}^{n} f\left(x_{i}\right)=\sum_{\left\{i_{1}, \ldots, i_{m}\right\} \in P_{m}} f\left(\sum_{k=1}^{m} x_{i_{k}}\right),
$$

for all $x_{1}, x_{2}, \ldots, x_{n} \in X$ if and only if $f_{e}$ satisfies the quadratic functional equation

$$
f(x+y)+f(x-y)=2 f(x)+2 f(y) \text { for all } x, y \in X,
$$

and $f_{o}$ satisfies the Cauchy functional equation

$$
f(x+y)=f(x)+f(y) \quad \text { for all } x, y \in X .
$$

Proof. To prove the necessity, suppose that a function $f: X \rightarrow Y$ satisfies (1). We will show that $f_{e}$ satisfies (2) and $f_{o}$ satisfies (3).

Putting $\left(x_{1}, x_{2}, \ldots, x_{n}\right)=(0,0, \ldots, 0)$ in $(1)$, we obtain

$$
\left(\begin{array}{c}
n-2 \\
m-2
\end{array}\right) f(0)+\left(\begin{array}{c}
n-2 \\
m-1
\end{array}\right) n f(0)=\left(\begin{array}{l}
n \\
m
\end{array}\right) f(0) .
$$

It can be verified that $\left(\begin{array}{c}n-2 \\ m-2\end{array}\right)+n\left(\begin{array}{c}n-2 \\ m-1\end{array}\right)>\left(\begin{array}{l}n \\ m\end{array}\right)$ for all integers $m$ and $n$ with $1<m<n$. Thus, $f(0)=0$. Putting $\left(x_{1}, x_{2}, \ldots, x_{n}\right)=(x, y,-y, 0,0, \ldots, 0)$ in $(1)$ and taking 
into account the fact that $f(0)=0$, we obtain

$$
\begin{aligned}
\left(\begin{array}{l}
n-2 \\
m-2
\end{array}\right) & f(x)+\left(\begin{array}{l}
n-2 \\
m-1
\end{array}\right)(f(x)+f(y)+f(-y)) \\
= & \left(\begin{array}{l}
n-3 \\
m-3
\end{array}\right) f(x)+\left(\begin{array}{c}
n-3 \\
m-2
\end{array}\right)(f(x+y)+f(x-y)) \\
& +\left(\begin{array}{c}
n-3 \\
m-1
\end{array}\right)(f(x)+f(y)+f(-y)),
\end{aligned}
$$

which simplifies to

$$
2 f(x)+f(y)+f(-y)=f(x+y)+f(x-y) \text { for all } x, y \in X .
$$

Replacing $x$ and $y$ in (5) with $-x$ and $-y$, respectively, we obtain

$$
2 f(-x)+f(-y)+f(y)=f(-x-y)+f(y-x) \text { for all } x, y \in X .
$$

Taking half the sum of (5) and (6), we obtain

$$
2 f_{e}(x)+2 f_{e}(y)=f_{e}(x+y)+f_{e}(x-y) \text { for all } x, y \in X,
$$

which shows that $f_{e}$ satisfies (2). Taking half the difference of (5) and (6), we obtain

$$
2 f_{o}(x)=f_{o}(x+y)+f_{o}(x-y) \quad \text { for all } x, y \in X,
$$

which is recognized as the Jensen functional equation. Noting that $f_{o}(0)=0$, we can verify that $f_{o}$ satisfies (3).

To prove the sufficiency, suppose that the even part and the odd part of a function $f: X \rightarrow Y$ satisfy (2) and (3), respectively. We need to show that $f$ satisfies (1). It should be noted that a linear combination of two solutions of (1) yields just another solution; therefore, it is sufficient to prove that both $f_{e}$ and $f_{o}$ satisfy (1).

First consider the odd part, $f_{o}$, and make use of the linearity of the Cauchy functional equation. The left-hand side of (1) becomes

$$
\begin{aligned}
\left(\begin{array}{l}
n-2 \\
m-2
\end{array}\right) f_{o}\left(\sum_{i=1}^{n} x_{i}\right)+\left(\begin{array}{l}
n-2 \\
m-1
\end{array}\right) \sum_{i=1}^{n} f_{o}\left(x_{i}\right) \\
=\left(\begin{array}{l}
n-2 \\
m-2
\end{array}\right) \sum_{i=1}^{n} f_{o}\left(x_{i}\right)+\left(\begin{array}{l}
n-2 \\
m-1
\end{array}\right) \sum_{i=1}^{n} f_{o}\left(x_{i}\right) \\
=\left(\begin{array}{l}
n-1 \\
m-1
\end{array}\right) \sum_{i=1}^{n} f_{o}\left(x_{i}\right)
\end{aligned}
$$

and the right-hand side of (1) becomes

$$
\sum_{\left\{i_{1}, \ldots, i_{m}\right\} \in P_{m}} f_{o}\left(\sum_{k=1}^{m} x_{i_{k}}\right)=\sum_{\left\{i_{1}, \ldots, i_{m}\right\} \in P_{m}} \sum_{k=1}^{m} f_{o}\left(x_{i_{k}}\right) .
$$


Expanding the sum on the right-hand side and collecting the terms,

$$
\sum_{\left\{i_{1}, \ldots, i_{m}\right\} \in P_{m}} f_{o}\left(\sum_{k=1}^{m} x_{i_{k}}\right)=\frac{m}{n}\left(\begin{array}{c}
n \\
m
\end{array}\right) \sum_{i=1}^{n} f_{o}\left(x_{i}\right)=\left(\begin{array}{c}
n-1 \\
m-1
\end{array}\right) \sum_{i=1}^{n} f_{o}\left(x_{i}\right) .
$$

Thus, we have established (1) on the odd part of $f$.

For the even part, it can be proved by mathematical induction (see, for example, [3]) that

$$
f_{e}\left(\sum_{i=1}^{n} x_{i}\right)+(n-2) \sum_{i=1}^{n} f_{e}\left(x_{i}\right)=\sum_{1 \leq i<j \leq n} f_{e}\left(x_{i}+x_{j}\right)
$$

for all integers $n$. For any integers $m$ and $n$ with $1<m<n$, the $m$-dimensional case of (9) with variables $x_{i_{1}}, x_{i_{2}}, \ldots, x_{i_{m}}$ is

$$
f_{e}\left(\sum_{k=1}^{m} x_{i_{k}}\right)+(m-2) \sum_{k=1}^{m} f_{e}\left(x_{i_{k}}\right)=\sum_{1 \leq k<l \leq m} f_{e}\left(x_{i_{k}}+x_{i_{l}}\right) .
$$

Summing the above equation for all $\left\{x_{i_{1}}, x_{i_{2}}, \ldots, x_{i_{m}}\right\} \subset\left\{x_{1}, x_{2}, \ldots, x_{n}\right\}$,

$$
\begin{gathered}
\sum_{\left\{i_{1}, \ldots, i_{m}\right\} \subset P_{m}} f_{e}\left(\sum_{k=1}^{m} x_{i_{k}}\right)+(m-2)\left(\begin{array}{c}
n-1 \\
m-1
\end{array}\right) \sum_{i=1}^{n} f_{e}\left(x_{i}\right) \\
=\left(\begin{array}{c}
n-2 \\
m-2
\end{array}\right) \sum_{1 \leq i<j \leq n} f_{e}\left(x_{i}+x_{j}\right) .
\end{gathered}
$$

Finally, eliminating $\sum_{1 \leq i<j \leq n} f\left(x_{i}+x_{j}\right)$ from (9) and (10),

$$
\left(\begin{array}{l}
n-2 \\
m-2
\end{array}\right) f_{e}\left(\sum_{i=1}^{n} x_{i}\right)+\left(\begin{array}{c}
n-2 \\
m-1
\end{array}\right) \sum_{i=1}^{n} f_{e}\left(x_{i}\right)=\sum_{\left\{i_{1}, \ldots, i_{m}\right\} \in P_{m}} f_{e}\left(\sum_{k=1}^{m} x_{i_{k}}\right),
$$

which shows that $f_{e}$ satisfies (1).

Thus, $f$ satisfies (1) and the proof is complete.

\section{The generalized stability}

The following theorem provides a general condition for which a true solution discussed in Theorem 1 exists near an approximate solution. For convenience, we define

$$
\begin{aligned}
& D_{m} f\left(x_{1}, \ldots, x_{n}\right) \\
& \quad=\left(\begin{array}{l}
n-2 \\
m-2
\end{array}\right) f\left(\sum_{i=1}^{n} x_{i}\right)+\left(\begin{array}{l}
n-2 \\
m-1
\end{array}\right) \sum_{i=1}^{n} f\left(x_{i}\right)-\sum_{\left\{i_{1}, \ldots, i_{m}\right\} \subset P_{m}} f\left(\sum_{k=1}^{m} x_{i_{k}}\right),
\end{aligned}
$$

for any integers $m$ and $n$ with $1<m<n$. 
THEOREM 2. Let $1<m<n$ be integers, $X$ be a real vector space, $Y$ be a Banach space and $\phi: X^{n} \rightarrow[0, \infty)$ be an even function with respect to each variable. Define $\varphi(x)=\phi(x, x,-x, 0, \ldots, 0)$ for all $x \in X$. If

$$
\left\{\begin{array}{l}
\sum_{i=0}^{\infty} 2^{-i} \varphi\left(2^{i} x\right) \quad \text { converges for all } x \in X, \text { and } \\
\lim _{s \rightarrow \infty} 2^{-s} \phi\left(2^{s} x_{1}, \ldots, 2^{s} x_{n}\right)=0 \text { for all } x_{1}, \ldots, x_{n} \in X,
\end{array}\right.
$$

or

$$
\left\{\begin{array}{l}
\sum_{i=1}^{\infty} 4^{i} \varphi\left(2^{-i} x\right) \quad \text { converges for all } x \in X, \text { and } \\
\lim _{s \rightarrow \infty} 4^{s} \phi\left(2^{-s} x_{1}, \ldots, 2^{-s} x_{n}\right)=0 \quad \text { for all } x_{1}, \ldots, x_{n} \in X,
\end{array}\right.
$$

and a function $f: X \rightarrow Y$ satisfies

$$
\left\|D_{m} f\left(x_{1}, \ldots, x_{n}\right)\right\| \leq \phi\left(x_{1}, \ldots, x_{n}\right) \quad \text { for all } x_{1}, \ldots, x_{n} \in X
$$

then there exists a unique function $T: X \rightarrow Y$ that satisfies (1) and, for all $x \in X$,

$$
\begin{aligned}
\| f(x)+ & p f(0)-T(x) \| \\
& \leq \begin{cases}\frac{1}{2} \sum_{i=0}^{\infty} 2^{-i} \varphi\left(2^{i} x\right)+\frac{1}{4} \sum_{i=0}^{\infty} 4^{-i} \varphi\left(2^{i} x\right) & \text { if (12) holds } \\
\frac{1}{2} \sum_{i=1}^{\infty} 2^{i} \varphi\left(2^{-i} x\right)+\frac{1}{4} \sum_{i=1}^{\infty} 4^{i} \varphi\left(2^{-i} x\right) & \text { if (13) holds }\end{cases}
\end{aligned}
$$

where $p=(((n-1)(n-2)) /(3 m))-1$. The function $T$ is given by

$$
T(x)= \begin{cases}\lim _{s \rightarrow \infty} 2^{-s} f_{o}\left(2^{s} x\right)+4^{-s} f_{e}\left(2^{s} x\right) & \text { if (12) holds } \\ \lim _{s \rightarrow \infty} 2^{s} f_{o}\left(2^{-s} x\right)+4^{s} f_{e}\left(2^{-s} x\right) & \text { if (13) holds }\end{cases}
$$

for all $x \in X$.

PROOF. We will first prove the theorem for a function $\phi$ satisfying (12). Putting $\left(x_{1}, x_{2}, \ldots, x_{n}\right)=(x, x,-x, 0,0, \ldots, 0)$ in (14) and simplifying,

$$
\|3 p f(0)+3 f(x)+f(-x)-f(2 x)\| \leq \varphi(x),
$$

where $p$ is defined as in the theorem. Replacing $x$ in the above equation by $-x$,

$$
\|3 p f(0)+3 f(-x)+f(x)-f(-2 x)\| \leq \varphi(-x)=\varphi(x) .
$$

From (17) and (18), we infer that, for all $x \in X$,

$$
\left\|3 p f(0)+4 f_{e}(x)-f_{e}(2 x)\right\| \leq \varphi(x),
$$


and

$$
\left\|2 f_{o}(x)-f_{o}(2 x)\right\| \leq \varphi(x) .
$$

Define a function $g_{e}: X \rightarrow Y$ by

$$
g_{e}(x)=f_{e}(x)+p f(0) \text { for all } x \in X .
$$

Then (19) becomes

$$
\left\|4 g_{e}(x)-g_{e}(2 x)\right\| \leq \varphi(x)
$$

which can be rewritten as

$$
\left\|g_{e}(x)-4^{-1} g_{e}(2 x)\right\| \leq 4^{-1} \varphi(x) .
$$

For each positive integer $s$,

$$
\begin{aligned}
\left\|g_{e}(x)-4^{-s} g_{e}\left(2^{s} x\right)\right\| & =\left\|\sum_{i=0}^{s-1}\left(4^{-i} g_{e}\left(2^{i} x\right)-4^{-(i+1)} g_{e}\left(2^{i+1} x\right)\right)\right\| \\
& \leq \sum_{i=0}^{s-1} 4^{-i}\left\|\left(g_{e}\left(2^{i} x\right)-4^{-1} g_{e}\left(2 \cdot 2^{i} x\right)\right)\right\| \\
& \leq \frac{1}{4} \sum_{i=0}^{s-1} 4^{-i} \varphi\left(2^{i} x\right) .
\end{aligned}
$$

Similarly, we can show that, for every integer $s$,

$$
\left\|f_{o}(x)-2^{-s} f_{o}\left(2^{s} x\right)\right\| \leq \frac{1}{2} \sum_{i=0}^{s-1} 2^{-i} \varphi\left(2^{i} x\right) .
$$

The convergence of the sequence $\left\{4^{-s} g_{e}\left(2^{s} x\right)\right\}$ can be settled as follows. For every positive integer $t$,

$$
\begin{aligned}
\left\|4^{-s} g_{e}\left(2^{s} x\right)-4^{-(s+t)} g_{e}\left(2^{s+t} x\right)\right\| & =4^{-s}\left\|g_{e}\left(2^{s} x\right)-4^{-t} g_{e}\left(2^{t} \cdot 2^{s} x\right)\right\| \\
& \leq 4^{-s} \cdot \frac{1}{4} \sum_{i=0}^{t-1} 4^{-i} \varphi\left(2^{i} \cdot 2^{s} x\right) \\
& \leq \frac{1}{4} \sum_{i=0}^{\infty} 4^{-(i+s)} \varphi\left(2^{i+s} x\right)
\end{aligned}
$$

From (12), we know that $\sum_{i=0}^{\infty} 4^{-(i+s)} \varphi\left(2^{i+s} x\right) \leq \sum_{i=0}^{\infty} 4^{-i} \varphi\left(2^{i} x\right)$ converges; so, it follows that $\lim _{s \rightarrow \infty}(1 / 4) \sum_{i=0}^{\infty} 4^{-(i+s)} \varphi\left(2^{i+s} x\right)=0$. Therefore, we have a Cauchy sequence in a Banach space. Let

$$
T_{e}(x)=\lim _{s \rightarrow \infty} 4^{-s} g_{e}\left(2^{s} x\right)=\lim _{s \rightarrow \infty} 4^{-s} f_{e}\left(2^{s} x\right) \quad \text { for all } x \in X .
$$


Thus,

$$
\left\|g_{e}(x)-T_{e}(x)\right\| \leq \frac{1}{4} \sum_{i=0}^{\infty} 4^{-i} \varphi\left(2^{i} x\right) .
$$

Similarly, the inequality on $f_{e}$ leads us to

$$
T_{o}(x)=\lim _{s \rightarrow \infty} 2^{-s} f_{o}\left(2^{s} x\right) \quad \text { for all } x \in X,
$$

and

$$
\left\|f_{o}(x)-T_{o}(x)\right\| \leq \frac{1}{2} \sum_{i=0}^{\infty} 2^{-i} \varphi\left(2^{i} x\right) .
$$

If we define a function $T: X \rightarrow Y$ by

$$
T(x)=T_{o}(x)+T_{e}(x) \quad \text { for all } x \in X,
$$

then

$$
\begin{aligned}
\|f(x)+p f(0)-T(x)\| & \leq\left\|f_{o}(x)-T_{o}(x)\right\|+\left\|g_{e}(x)-T_{e}(x)\right\| \\
& \leq \frac{1}{2} \sum_{i=0}^{\infty} 2^{-i} \varphi\left(2^{i} x\right)+\frac{1}{4} \sum_{i=0}^{\infty} 4^{-i} \varphi\left(2^{i} x\right) .
\end{aligned}
$$

In order to show that $T$ satisfies (1), we will prove that the even part and the odd part of $T$ satisfy (1). Define the even part and the odd part of $D_{m} f$ by

$$
\begin{aligned}
D_{m} f_{e}\left(x_{1}, \ldots, x_{n}\right) & =\frac{D_{m} f\left(x_{1}, \ldots, x_{n}\right)+D_{m} f\left(-x_{1}, \ldots,-x_{n}\right)}{2} \\
D_{m} f_{o}\left(x_{1}, \ldots, x_{n}\right) & =\frac{D_{m} f\left(x_{1}, \ldots, x_{n}\right)-D_{m} f\left(-x_{1}, \ldots,-x_{n}\right)}{2} .
\end{aligned}
$$

For a positive integer $s$ and for all $x_{1}, x_{2}, \ldots, x_{n} \in X$,

$$
\begin{aligned}
\left\|D_{m} f_{e}\left(2^{s} x_{1}, \ldots, 2^{s} x_{n}\right)\right\| \leq & \frac{1}{2}\left\|D_{m} f\left(2^{s} x_{1}, \ldots, 2^{s} x_{n}\right)\right\| \\
& +\frac{1}{2}\left\|D_{m} f\left(-2^{s} x_{1}, \ldots,-2^{s} x_{n}\right)\right\| \\
\leq & \phi\left(2^{s} x_{1}, \ldots, 2^{s} x_{n}\right) .
\end{aligned}
$$

If we divide the above inequality by $4^{s}$ and take the limit as $s \rightarrow \infty$, then the righthand side vanishes according to (12) and we obtain from the definition of $T_{e}$ that

$$
\left(\begin{array}{c}
n-2 \\
m-2
\end{array}\right) T_{e}\left(\sum_{i=1}^{n} x_{i}\right)+\left(\begin{array}{c}
n-2 \\
m-1
\end{array}\right) \sum_{i=1}^{n} T_{e}\left(x_{i}\right)=\sum_{\left\{i_{1}, \ldots, i_{m}\right\} \in P_{m}} T_{e}\left(\sum_{k=1}^{m} x_{i_{k}}\right),
$$

for all $x_{1}, x_{2}, \ldots, x_{n} \in X$. We can similarly show that $T_{o}$ satisfies (1). Hence, $T=T_{e}+T_{o}$ satisfies (1). 
To prove the uniqueness of $T$, suppose there exists another function $T^{\prime}: X \rightarrow Y$ such that $T^{\prime}$ satisfies (1) and (15). We have proved in Theorem 1 that $T_{e}$ satisfies the quadratic functional equation (2) and $T_{o}$ satisfies the Cauchy functional equation (3); therefore, $T_{e}(r x)=r^{2} T_{e}(x)$ and $T_{o}(r x)=r T_{o}(x)$ for every rational number $r$ and for every $x \in X$. Thus,

$$
\left\|T(x)-T^{\prime}(x)\right\| \leq\left\|T_{e}(x)-T_{e}^{\prime}(x)\right\|+\left\|T_{o}(x)+T_{o}^{\prime}(x)\right\| .
$$

For any positive integer $s$ and for each $x \in X$,

$$
\begin{aligned}
\left\|T_{e}(x)-T_{e}^{\prime}(x)\right\| & =4^{-s}\left\|T_{e}\left(2^{s} x\right)-T_{e}^{\prime}\left(2^{s} x\right)\right\| \\
& \leq 4^{-s}\left\|g_{e}\left(2^{s} x\right)-T_{e}\left(2^{s} x\right)\right\|+4^{-s}\left\|g_{e}\left(2^{s} x\right)-T_{e}^{\prime}\left(2^{s} x\right)\right\| \\
& \leq 2 \cdot 4^{-s} \cdot \frac{1}{4} \sum_{i=0}^{\infty} 4^{-i} \varphi\left(2^{i} \cdot 2^{s} x\right) \\
& =\frac{1}{2} \sum_{i=0}^{\infty} 4^{-(i+s)} \varphi\left(2^{i+s} x\right) .
\end{aligned}
$$

Taking the limit as $s \rightarrow \infty$, we have $\left\|T_{e}(x)-T_{e}^{\prime}(x)\right\| \leq 0$. Thus $T_{e}(x)=T_{e}^{\prime}(x)$ for all $x \in X$. Similarly, we can show that $T_{o}(x)=T_{o}^{\prime}(x)$ for all $x \in X$. Hence, $T(x)=T^{\prime}(x)$ for all $x \in X$.

The proof for the case when (13) holds can be done in a similar manner.

In the next few corollaries, we will give the stability of (1) in various senses. The following corollary proves the Hyers-Ulam stability.

Corollary 3. If a function $f: X \rightarrow Y$ satisfies

$$
\left\|D_{m} f\left(x_{1}, x_{2}, \ldots, x_{n}\right)\right\| \leq \varepsilon \text { for all } x_{1}, x_{2}, \ldots, x_{n} \in X
$$

for some $\varepsilon>0$, then there exists a unique function $T: X \rightarrow Y$ that satisfies (1) and

$$
\|f(x)+p f(0)-T(x)\| \leq \frac{4 \varepsilon}{3} \quad \text { for all } x \in X .
$$

PRoOF. Let $\phi\left(x_{1}, x_{2}, \ldots, x_{n}\right)=\varepsilon$ for all $x_{1}, x_{2}, \ldots, x_{n} \in X$ in Theorem 2. Hence, $\varphi(x)=\varepsilon$ for all $x \in X$. We can see that (12) holds. Therefore, it follows from the theorem that there exists a unique function $T: X \rightarrow Y$ such that

$$
\|f(x)+p f(0)-T(x)\| \leq \frac{1}{2} \sum_{i=0}^{\infty} 2^{-i} \varepsilon+\frac{1}{4} \sum_{i=0}^{\infty} 4^{-i} \varepsilon=\frac{4 \varepsilon}{3} \quad \text { for all } x \in X .
$$

The following corollary proves the Hyers-Ulam-Rassias stability of (1).

COROLlaRY 4. Let $p$ be a real number with $0<p<1$ or $p>2$. If a function $f: X \rightarrow Y$ satisfies

$$
\left\|D_{m} f\left(x_{1}, x_{2}, \ldots, x_{n}\right)\right\| \leq \varepsilon \sum_{i=1}^{n}\left\|x_{i}\right\|^{p} \quad \text { for all } x_{1}, x_{2}, \ldots, x_{n} \in X
$$


for some $\varepsilon>0$, then $f(0)=0$ and there exists a unique function $T: X \rightarrow Y$ that satisfies (1) and

$$
\|f(x)-T(x)\| \leq \frac{6 \varepsilon\left|3-2^{p}\right|}{\left(2-2^{p}\right)\left(4-2^{p}\right)}\|x\|^{p} \quad \text { for all } x \in X .
$$

PROOF. Substituting $x_{1}=x_{2}=\cdots=x_{n}=0$ into (21), we obtain

$$
\left(\begin{array}{l}
n-2 \\
m-2
\end{array}\right) f(0)+\left(\begin{array}{l}
n-2 \\
m-1
\end{array}\right) n f(0)=\left(\begin{array}{l}
n \\
m
\end{array}\right) f(0),
$$

as in (4). Thus, $f(0)=0$. Let $\phi\left(x_{1}, x_{2}, \ldots, x_{n}\right)=\varepsilon \sum_{i=1}^{n}\left\|x_{i}\right\|^{p}$ for all $x_{1}, x_{2}, \ldots, x_{n} \in X$. Then $\varphi(x)=3 \varepsilon\|x\|^{p}$ for all $x \in X$. If $0<p<1$, then (12) holds and it follows from Theorem 2 that

$$
\begin{aligned}
\|f(x)-T(x)\| & \leq \frac{1}{2} \sum_{i=0}^{\infty}\left(2^{-i} \cdot 3 \varepsilon\left\|2^{i} x\right\|^{p}\right)+\frac{1}{4} \sum_{i=0}^{\infty}\left(4^{-i} \cdot 3 \varepsilon\left\|2^{i} x\right\|^{p}\right) \\
& =\frac{3 \varepsilon}{2-2^{p}}\|x\|^{p}+\frac{3 \varepsilon}{4-2^{p}}\|x\|^{p} \\
& =\frac{6 \varepsilon\left(3-2^{p}\right)}{\left(2-2^{p}\right)\left(4-2^{p}\right)}\|x\|^{p} \quad \text { for all } x \in X .
\end{aligned}
$$

If $p>1$, then (13) holds, and we get a similar result.

For the generalized stability in the sense of Gavruta [1], we get a superstability of (1) when $n>3$ as stated in the following corollary.

COROLlary 5. Let $p_{1}, p_{2}, \ldots, p_{n} \geq 0$ and $r=\sum_{i=1}^{n} p_{i}$ with $0<r<1$ or $r>2$. If a function $f: X \rightarrow Y$ satisfies

$$
\left\|D_{m} f\left(x_{1}, x_{2}, \ldots, x_{n}\right)\right\| \leq \varepsilon \prod_{i=1}^{n}\left\|x_{i}\right\|^{p_{i}} \quad \text { for all } x_{1}, x_{2}, \ldots, x_{n} \in X .
$$

for some $\varepsilon>0$, then:

(1) if $n>3$, then $f$ satisfies equation (1); and

(2) if $n=3$, then there exists a unique function $T: X \rightarrow Y$ that satisfies equation (1) and

$$
\|f(x)-T(x)\| \leq \frac{\varepsilon\left|3-2^{r}\right|}{\left(2-2^{r}\right)\left(4-2^{r}\right)}\|x\|^{r} \quad \text { for all } x \in X .
$$

ProOF. We can show that $f(0)=0$ by the same substitution used in the proof of Corollary 4. Let $\phi\left(x_{1}, x_{2}, \ldots, x_{n}\right)=\varepsilon \prod_{i=1}^{n}\left\|x_{i}\right\|^{p_{i}}$ for all $x_{1}, x_{2}, \ldots, x_{n} \in X$. Then, for all $x \in X$,

$$
\varphi(x)= \begin{cases}0 & \text { if } n>3 \\ \varepsilon\|x\|^{r} & \text { if } n=3\end{cases}
$$


If $n>3$, then we can see that $f$ satisfies (1). If $n=3$, then we consider two cases: $0<r<1$ and $r>2$. If $0<r<1$, then (12) holds and for all $x \in X$, by Theorem 2,

$$
\begin{aligned}
\|f(x)-T(x)\| & \leq \frac{1}{2} \sum_{i=0}^{\infty}\left(2^{-i} \cdot \varepsilon\left\|2^{i} x\right\|^{r}\right)+\frac{1}{4} \sum_{i=0}^{\infty}\left(4^{-i} \cdot \varepsilon\left\|2^{i} x\right\|^{r}\right) \\
& =\frac{\varepsilon}{2-2^{r}}\|x\|^{r}+\frac{\varepsilon}{4-2^{r}}\|x\|^{r} \\
& =\frac{2 \varepsilon\left(3-2^{r}\right)}{\left(2-2^{r}\right)\left(4-2^{p}\right)}\|x\|^{r} .
\end{aligned}
$$

If $r>2$, then (13) holds and we get a similar result.

\section{References}

[1] P. Gavruta, 'A generalization of the Hyers-Ulam-Rassias stability of approximately additive mappings', J. Math. Anal. Appl. 184 (1994), 431-436.

[2] D. H. Hyers, 'On the stability of the linear functional equations', Proc. Natl. Acad. Sci. U.S.A. 27 (1941), 222-224.

[3] P. Nakmahachalasint, On the generalized Ulam-Gavruta-Rassias stability of mixed-type linear and Euler-Lagrange-Rassias functional equations. IJMMS (2007). Available at: http://www.hindawi.com/GetArticle.aspx?doi=10.1155/2007/63239\&e=cta.

[4] J. M. Rassias, 'On the Ulam stability of mixed type mappings on restricted domains', J. Math. Anal, Appl. 276 (2002), 747-762.

[5] Th. M. Rassias, 'On the stability of the linear mapping in Banach spaces', Proc. Amer. Math. Soc. 72 (1978), 297-300.

[6] S. M. Ulam, Problems in modern mathematics (Wiley, New York, 1964), Ch. 6.

\section{Department of Mathematics}

Faculty of Science

Chulalongkorn University

Bangkok 10330

Thailand

e-mail: Paisan.N@chula.ac.th 